\title{
THEORY OF NEAR-ZONE MEASUREMENT WITH TRANSFORMATION TO AERIAL SURFACE AND GAIN MEASUREMENT
}

\author{
Ján Ivanka * Hana Chudá ${ }^{* *}$
}

\begin{abstract}
The paper presents an analysis study of the theory of measurement in the near, Fresnel and Fraunhofer zone with transformation to aerial surface as well as real measurements of the gain of aerial system of electromagnetic fields of devices and components used in the safety industry in an aneochoic chamber.

K e y w o r ds: electromagnetical fields, electromagnetic compatibility, measurements
\end{abstract}

\section{INTRODUCTION}

The goal of this report is to give you an overview of advantages and disadvantages of the near zone measurement process and comparison with measurements performed in distant zone. Probes with low gain offer advantages from the calculation point of view, because is often not necessary to perform compensation of the probe during calculation. That is true mostly for cylindrical and spherical monitoring/scanning (spherical monitoring is even less sensitive to the probe influence then cylindrical monitoring). Using probes with two orthogonally polarized signals, to ensure monitoring/scanning of two data files during one scan, will shorten the time to half, which is necessary for data collection in comparison with traditional monitoring/scanning with one output probe. This paper gives you several typical samples of probes with one or two outputs for reception of orthogonally polarized signals. Most samples apply to probes with low gain (approx. $7 \mathrm{~dB}$ ). For any measuring method, one of the most essential requirements is reliable estimate of measurement errors. This is mostly true for antenna measurement methods in near zone. The determination of error limits for any measuring system for applicable combination of antenna/probe/near zone may be difficult and time consuming, whereas the mathematical complexity is the main reason for the difficulty. There is an entire range of error sources some of which are caused by the probe. That is why it is important, during the probe specification, to consider the essential requirements that are expected from the probes.

Often experiments to skip the mathematics are performed in order to set the error limits for general measuring technique, using measurements for applicable antenna. During this approach, measuring results in the distant and the near zone are compared and the differences between these two methods are considered as the error scale/criteria for measurements done in the near zone. This approach however, has several shortcomings. Firstly, the observed differences are partially due to the error influence in the distant zone. Plus it is difficult to generalize results for other antenna or measuring system. The most crucial measuring parameters or contributing elements from each error source cannot be determined and end measurement in distant zone may be impractical for some types of antennas, which are suitable for measuring in the near zone. That means that such comparisons have no value. They demonstrate reliability, help to establish credibility without detailed mathematical analysis and point out areas where more thorough and detail analysis needs to be done.

They are one of the parts of the error analysis but in no case they represent the entire picture. The entire and general analysis requires combination of several approaches, analytical and experimental, in order to identify all possible error sources and also their contributions to the final calculation results could be estimated.

Auxiliary antenna (probe) must be selected carefully because suitably selected antenna may improve the precision of the measuring process dramatically, because the probe works as a space phase filter in the near zone and also as space spectrum filter in the distant zone. It is possible to select probes with low gains, because the need to compensate the probe during calculation is often not necessary. Or you may choose probe with higher gains that offer wider variety of advantages mainly during measurements performed on flat surface. This report analyzes requirements that are expected from probes used for near field measurements and also includes advantages and disadvantages of each solution. Great deal of attention is given to probes with two outputs for orthogonally polarized signals, which may lower the measuring time by half. More detailed analysis of errors influenced by the probe will be included in report that shall focus on the influence

\footnotetext{
* Department of Security Engineering, ${ }^{* *}$ Department of Mathematics, Faculty of Applied Informatics, Tomas Bata University in Zlín, T.G. Masaryka 5555, 76001 Zlín, Czech Republic, ivanka@fai.utb.cz, chuda@fai.utb.cz
} 
of environment and measuring instruments errors on the measuring results performed in near zone.

\section{THEORETICAL PART}

Electromagnetic compatibility (EMC) represents a new science discipline that had occurred as lately as during the sixties, and over a relatively long period of time, it had been known within only a narrow circle of experts involved in the military and cosmic space research. It can be defined as the ability of a device or system to work in the given electromagnetic environment without generating inadmissible electromagnetic interference with the devices and instruments located in such environment. EMC therefore represents the ability of concurrent proper functioning, i.e. the coexistence of devices or systems (both electronic or mechanical with integrated electronics and biological), which are sharing the same electromagnetic environment without affecting each other in respect of their normal functions. It therefore serves as the indicator of quality and reliability of the equipment. For real measurements in the near zone of the system components used in the commercial safety industry by means of a Hall probe, there have studies with detailed theoretical relationships been prepared, which are important for the numerical processing of each particular measurement.

To be able to specify precisely the aerial characteristics, we need to know $R^{\prime}(\mathbf{K})$ values. This is particularly important when the polarization characteristics of the probe are not good enough. To solve the equations (1) and those for $I_{o}^{\prime \prime}(\mathbf{K})$, components can be written down

$$
\begin{aligned}
& R_{1}^{\prime}(\mathbf{K}) T_{1}(\mathbf{K})+R_{2}^{\prime}(\mathbf{K}) T_{2}(\mathbf{K})=I_{0}^{\prime}(\mathbf{K}) \\
& R_{1}^{\prime \prime}(\mathbf{K}) T_{1}(\mathbf{K})+R_{2}^{\prime \prime}(\mathbf{K}) T_{2}(\mathbf{K})=I_{0}^{\prime \prime}(\mathbf{K})
\end{aligned}
$$

where $R^{\prime}(\mathbf{K})=R_{1}^{\prime}(\mathbf{K}) x^{0}+R_{2}^{\prime}(\mathbf{K}) y^{0}, R^{\prime \prime}(\mathbf{K})=R_{1}^{\prime \prime}(\mathbf{K}) x^{0}+$ $R_{2}^{\prime \prime}(\mathbf{K}) y^{0}$ and $T(\mathbf{K})=T_{1}(\mathbf{K}) x^{0}+T_{2}(\mathbf{K}) y^{0}$. It is possible to use components that are perpendicular to the direction of propagation $t_{m}=t_{m}(\mathbf{K})$ for the basic polarization of the measured aerial, and $t_{c}=t_{c}(\mathbf{K})$ for the cross polarization and similarly for $t_{m}=t_{m}(\mathbf{K})$ and $t_{c}=t_{c}(\mathbf{K})$.

We will then receive fully analogical equations

$$
\begin{gathered}
t_{m}=\frac{1}{\Delta}\left(I^{\prime} r_{c}^{\prime \prime}-I^{\prime \prime} r_{c}^{\prime}\right), \quad t_{c}=\frac{1}{\Delta}\left(I^{\prime \prime} r_{m}^{\prime}-I^{\prime} r_{m}^{\prime \prime}\right), \\
\Delta=r_{c}^{\prime \prime} r_{m}^{\prime}-r_{c}^{\prime} r_{m}^{\prime \prime} .
\end{gathered}
$$

Generally, probes should be chosen so that the basic component of the probe would be linked to the basic component of the measured aerial while the other probe should be linked to the cross component. This however cannot be applied to the measurement of circular polarization, nevertheless it can be assumed that $\left|r_{m}^{\prime}\right| \geq\left|r_{c}^{\prime}\right|$ and $\left|r_{c}^{\prime \prime}\right| \geq\left|r_{m}^{\prime \prime}\right|$.

Solution can be then written as follows

$$
t_{m}=\frac{\frac{I^{\prime}}{r_{m}^{\prime}}-\frac{I^{\prime \prime}}{r_{c}^{\prime \prime}} \varrho_{r}^{\prime}}{1-\frac{\varrho_{r}^{\prime}}{\varrho_{r}^{\prime \prime}}}, \quad t_{c}=\frac{\frac{I^{\prime \prime}}{r_{c}^{\prime \prime}}-\frac{I^{\prime}}{r_{m}^{\prime} \varrho_{r}^{\prime \prime}}}{1-\frac{\varrho_{r}^{\prime}}{\varrho_{r}^{\prime \prime}}},
$$

where the receiving polarization relationships are

$$
\varrho_{r}^{\prime}(\mathbf{K})=\frac{r_{c}^{\prime}(\mathbf{K})}{r_{m}^{\prime}(\mathbf{K})}, \quad \varrho_{r}^{\prime \prime}(\mathbf{K})=\frac{r_{c}^{\prime \prime}(\mathbf{K})}{r_{m}^{\prime \prime}(\mathbf{K})}
$$

The upper equation is applicable on any orthogonal system of coordinates. Equations (2) and (3) represent two versions of the equations for the probe correction. To be able to solve equation (1), spectra $\mathbf{R}^{\prime}$ and $\mathbf{R}^{\prime \prime}$ must be linearly independent. It follows from (3) that

$$
\varrho_{r}^{\prime}(\mathbf{K}) \neq \varrho_{r}^{\prime \prime}(\mathbf{K})
$$

where dependence on $\mathbf{K}$ should explicitly emphasize that this condition must be fulfilled for each direction requiring calculation of $\boldsymbol{t}(\mathbf{K})$. Requirement for linear independence does not mean that the probes should be orthogonally polarized, although it would be the best arrangement scheme. Nor should it eliminate the use of circularly polarized probes. If the first of them is polarized clockwise and the other one counter-clockwise, requirement of (5) will thereby be fulfilled. In many cases only one probe is used instead of two different probes. The probe is linearly polarized and the "second" one can be obtained by turning the probe by $90^{\circ}$ around its axis. One probe measurement will then be sufficient enough.

Equation of transmission and probe correction was obtained for an option of transmitting measured aerial and receiving probe. However, a converse option with transmitting probe is also possible. In such a case, measurement and calculation will be done in parallel to the option using transmitting measured aerial.

If the aerial is reciprocal then $\boldsymbol{t}$ and $\boldsymbol{r}$ bound by the reciprocity equation, gain $G(\mathbf{K})$ and effective area of the aerial $A_{e}(\mathbf{K})$ will be

$$
\begin{aligned}
\eta k \mathbf{r}(\mathbf{K}) & =\gamma Y_{0} \boldsymbol{t}(-\mathbf{K}), \\
G(\mathbf{K}) & =\frac{4 \pi Y_{0} \gamma^{2}|\boldsymbol{t}(\mathbf{K})|^{2}}{\eta_{0}\left(1-\left|\Gamma_{a}\right|^{2}\right)} \\
A_{e}(\mathbf{K}) & =\frac{4 \pi^{2} \eta_{0}|\mathbf{r}(\mathbf{K})|^{2}}{Y_{0}\left(1-\left|\Gamma_{a}\right|^{2}\right)},
\end{aligned}
$$

where $Y_{0}=(\varepsilon / \mu)$ is wave admittance, $\eta_{0}$ represents characteristic admittance of the transmission line connected to the aerial and $\Gamma_{a}$ is aerial reflection coefficient. For TE waves, $\eta_{T E}$ is equal to $\frac{Y_{0} \gamma}{k}$ and for TM waves, $\eta_{T M}=\frac{Y_{0} k}{\gamma}$. In calculations relating to the measurement, use of both $Y_{0}$ and $\eta_{0}$ will be rare as they are mostly cancelled out in the final result. They should only be used if different lines are used for the aerial and probe. Relationships (6) to (8) are applicable to both aerial and probe. It is interesting to note that similar relationships can be applied even in more general setting, in which much more general-purpose environment types including chiral environment are considered. Similarly, both "transverse" 


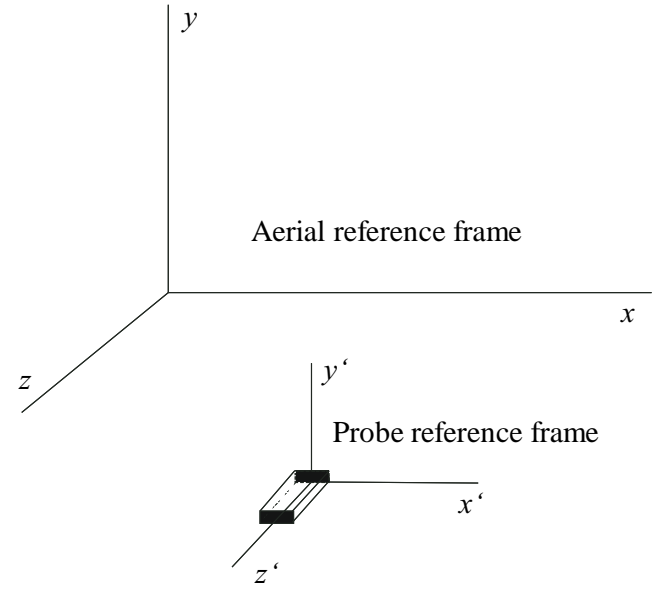

Fig. 1. Systems of coordinates of the measured aerial and probe

transmission coefficients $\mathbf{T}(\mathbf{K})$ and "general" transmission coefficients $\boldsymbol{t}(\mathbf{K})$ can be considered. Unfortunately, symbols used in the literature quoted herein differ from each other and the terminology itself is far from being unified (even if used by the same authors). This naturally does not contribute to understanding and legibility.

To be able to use the measurement data accurately and properly, system of coordinates of the probe should be clearly defined. Vector components $\mathbf{r}(\mathbf{K})$ as well as $\mathbf{K}$ should be defined considering the system of coordinates of the probe. The system of coordinates should therefore be defined unambiguously and all adequate means should be made available to carry out the probe settings for the near-zone measurement.

For a single output probe, $x$-axis is denoted by reference marks on two narrow-side walls of the waveguide, and similarly $y$-axis is denoted by reference marks on the broad-side walls of the waveguide. $Z$-axis is defined as the parallel to the broad and narrow inside walls of the waveguide. To ensure orientation of $z$-axis to be the same like for the near-zone measurement, the $\boldsymbol{z}$-axis must be oriented towards the probe interior, while for the majority of other applications it is defined as oriented away from the aerial. This definition is stable and accurate, however it should be pointed out that it may not be the most favourable for the setting process. Therefore $z$ direction may be measured with respect to the waveguide flange and the probe setting will be done by means of the flange surface, whereat an angle between $z$-axis and line perpendicular to the flange on $y z$ plane $\left(E_{0}\right.$ angle) and a similar angle on $x z$ plane $\left(A_{0}\right.$ angle) must be defined. Both of them, ideally, will be zero angles, which can often be achieved by mechanical working the flange surface. For a probe with two outputs, two ports $(X$ and $Y$ ) will be defined, and $x$ and $y$ ports will be defined using two sets of reference marks at the open end of the probe.

$Z$-axis is defined as the perpendicular to the plane of aperture towards the interior of the probe.

In addition to rectangular $0 x z y$ systems, definition of spherical systems firmly linked to the aerial is required in order to determine directions in space and vector components by means of two spherical angles. There is a wide array of options and we will choose three thereof. In mathematics, the most common system is that with $z$-axis as well as and angles, which is shown in Fig. 2a. Figures $2 \mathrm{~b}$. and 2c. show systems with $A, E$ and $\alpha, \varepsilon$ angles. Respective direction in space can be specified using either propagation vector components $\boldsymbol{k}$ or whichever system of angles, be it $\theta, \varphi ; A, E$ or $\alpha, \varepsilon$. Similarly, vector transverse to the radial direction of the sphere (direction of propagation) can be figured using three systems of spherical components $\theta^{0}, \varphi^{0} ; A^{0}, E^{0}$ or $\alpha^{0}, \varepsilon^{0}$. Transformation of one system to another can be effected using the following relationships

$$
\begin{aligned}
& \frac{k_{x}}{k}=\sin \theta \cos \varphi=\sin A \cos E=\sin \alpha, \\
& \frac{k_{y}}{k}=\sin \theta \sin \varphi=\sin E=\cos \alpha \sin \varepsilon, \\
& \frac{k_{z}}{k}=\cos \theta=\cos A \cos E=\cos \alpha \cos \varepsilon .
\end{aligned}
$$

Transformations are usually required if the measurement in one system is prescribed $(e g, \theta, \varphi)$ while another system is to be used for final results $(e g, A, E)$.

The first step in the measurement process is the determination of polarization characteristics on an axis, which includes determination of gain, axial ratio, angle of inclination and sense of polarization for the probe and auxiliary aerial while using three-aerial method. Based on the
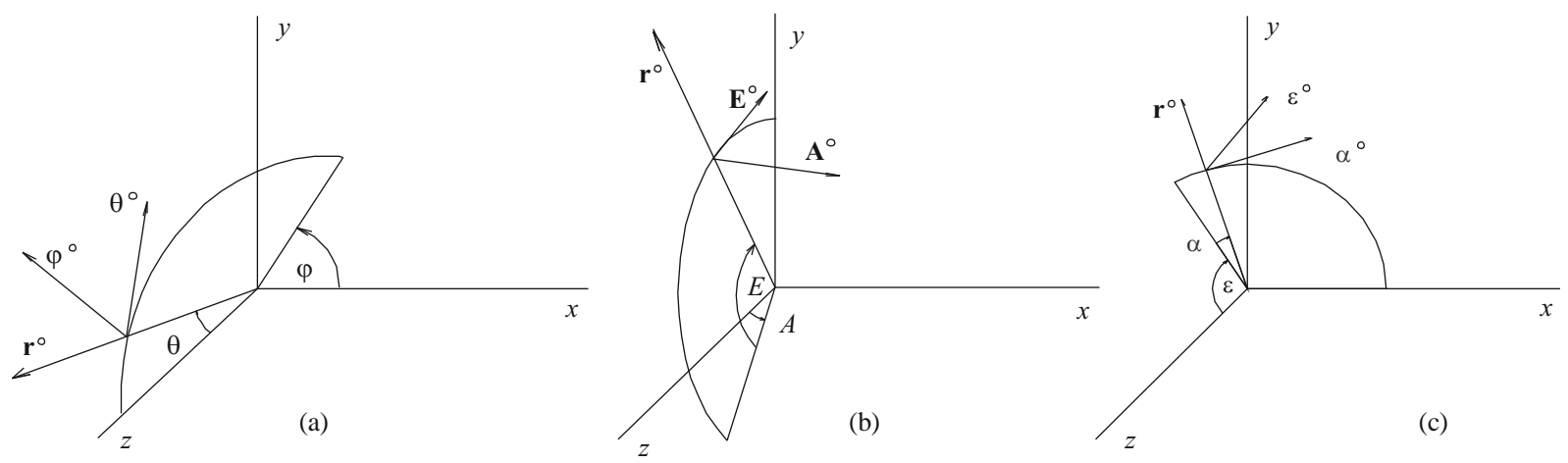

Fig. 2. Systems of coordinates used for calculation of measured values 
measurement of axial ratio $A$, angle of inclination $\tau$ and sense of polarization, circular polarization ratio $\rho_{r c}(0)$ on axis $(\mathbf{K}=0)$ shall be determined first:

$$
\rho_{r c}(0)=\frac{\left[r_{L}^{\prime}(0)\right]}{\left[r_{R}^{\prime}(0)\right]}
$$

using the following relationships:

$$
\left|\rho_{r c}(0)\right|=\left\{\begin{array}{c}
\frac{A(0)-1}{A(0)+1}, \text { for right-hand circular polarization, } \\
\frac{A(0)+1}{A(0)-1}, \text { for left-hand circular polarization, } \\
\operatorname{Arg}\left[\rho_{r c}(0)\right]=2 \tau(0)
\end{array}\right.
$$

wherefrom linear polarization ratio can be obtained as

$$
\left|\rho_{r l}(0)\right|=\frac{\left[r_{A}^{\prime}(0)\right]}{r_{E}^{\prime}(0)}=-j \frac{1+\rho_{r c}(0)}{1-\rho_{r c}(0)} .
$$

Sense of polarization is used to determine whether $\left|\rho_{r c}(0)\right|$ is higher or lower than the unit value $(=1)$ as this information cannot be obtained from the ratio of $A$ axes or angle of inclination $\tau$. Considering selected definition of coordinates, main component for the single-output probe as well as for the $Y$ port of the probe with two outputs will be the component $E$, having its component on the axis

$$
\left|r_{E}^{\prime}(0)\right|=\left|\frac{\left(1-\left|\Gamma_{p}\right|^{2}\right) Y_{0} G(0)}{4 \pi k^{2} \eta_{0}\left(1+\left|\rho_{s l}(0)\right|^{2}\right)}\right|^{\frac{1}{2}},
$$

where $G(0)$ is gain measured in direction on the axis, $\Gamma_{p}$ denotes reflection coefficient of the probe, $Y_{0}$ and $\eta_{0}$ are admittance coefficients that can be set to $Y_{0}=\eta_{0}$ in this particular case. Phase $r_{E}^{\prime}(0)$ can be chosen arbitrarily, and the size and phase of the transverse component can be obtained from (25). Components on the axes are

$$
\begin{aligned}
& r_{E}^{\prime}(0)=r_{\varphi}^{\prime}(0,0)=-r_{\theta}^{\prime}\left(0,-\frac{\pi}{2}\right) \\
& r_{A}^{\prime}(0)=r_{\varphi}^{\prime}\left(0,-\frac{\pi}{2}\right)=-r_{\theta}^{\prime}(0,0)
\end{aligned}
$$

Gain of aerial systems is measured using three basic methods (direct method, gain comparison method and three-aerial method). Basic equation for the determination of gain is (7). In all three methods, three values shall enter into the calculation of gain of the measured aerial system, namely measurement of relative values in the near zone (one or more sets), gain of standard aerial and one or more ratios of sizes and relative phase of transmission between the measuring input of the aerial and the output of probe such as $\frac{b_{0}^{\prime}(\mathbf{P})}{a_{0}}$. These three methods differ in how the standard of gain and number and type of measurements in the near zone and measurements of transmission are used.

The first method is based on direct use of basic equations and the probe is used as the gain standard. Data measured in the near zone relate to the input amplitudes and phases.
The second method is useful whenever neither the $i n$ sertion attenuation, nor the output measurement for the determination of $A^{\prime}$ and $A^{\prime \prime}$ are functional, or whenever the probe gain is unknown. When choosing $\mathbf{P}_{0}$ as the normalization point of the measured aerial and $\mathbf{P}_{2}$ for the standard aerial as well as indices $a p$ for the combination measured aerial - probe and $s p$ for the combination standard aerial — probe and $a$ for the measured aerial and $s$ for the standard aerial, the result for the probe adjusted by polarization to the measured and standard aerial will be

$$
\begin{aligned}
& G_{a}\left(\mathbf{K}_{0}\right)=G_{s}\left(\mathbf{K}_{0}\right) \times \\
& \frac{\left.\left.\left(1-\left|\Gamma_{s}\right|^{2}\right)\left|\delta_{x} \delta_{y} \sum_{i}{ }_{a p} B_{0}^{\prime}\left(\mathbf{P}_{i}\right) \exp \left(j \mathbf{K}_{0} \cdot \mathbf{P}_{i}\right)\right|^{2}\right|_{s} a_{0} a p b_{0}^{\prime}\left(\mathbf{P}_{0}\right)\right|^{2}}{\left.\left.\left(1-\left|\Gamma_{s}\right|^{2}\right)\left|\delta_{x} \delta_{y} \sum_{i}{ }_{s p} B_{0}^{\prime}\left(\mathbf{P}_{i}\right) \exp \left(j \mathbf{K}_{0} \cdot \mathbf{P}_{i}\right)\right|^{2}\right|_{a} a_{0} s p b_{0}^{\prime}\left(\mathbf{P}_{0}\right)\right|^{2}} .
\end{aligned}
$$

The third method uses two additional different probes in addition to the measured aerial and the near-zone detection is carried out for all three combinations. If measurement of insertion attenuation is done for all three combinations, we get three equations for the gain:

$$
\begin{aligned}
G_{a}(\mathbf{K}) G_{p 1}(0) & ={ }_{f} G_{a p 1}(\mathbf{K}), \\
G_{a}(\mathbf{K}) G_{p 2}(0) & ={ }_{f} G_{a p 2}(\mathbf{K}), \\
G_{p 1}(\mathbf{K}) G_{p 2}(0) & ={ }_{f} G_{p 1 p 2}(\mathbf{K}),
\end{aligned}
$$

Some values that have been measured using the above mentioned relationships and up-to-date program tool.

\section{EXPERIMENTAL PART}

\subsection{Probe compensation}

Probes with low gains are suitable from the calculation point of view, because the need to compensate the probe during the calculation is often not necessary. That is true mostly for cylindrical and spherical monitoring/scanning (spherical monitoring is even less sensitive to the probe influence then cylindrical monitoring). But these probes may have worse signal - noise ratio and must be sampled in distance that is set by the Nyguist sampling theory and demand better precision in the probe position settings. Probe with high gain $1525 \mathrm{~dB}$ receive energy under small angle in the distant zone and "will filter out" energy outside the axis. Also the probe in the near zone will deliver an average value of higher space frequencies that correspond with energy outside the axis. Probes with high gain are recommended if the corresponding field in the distant zone will be calculated for small angles (five degrees or less). Although these probes modify the radiating/emitting diagram, you may perform compensation of the radiating probe diagram.

Probes with high gains have four big advantages: 
Table 1. Axis measuring results of a typical probe with one output

\begin{tabular}{ccccc}
\hline $\begin{array}{c}\text { Frequency } \\
(\mathrm{GHz})\end{array}$ & $\begin{array}{c}\text { Gain } \\
(\mathrm{dB})\end{array}$ & $\begin{array}{c}\text { Axis ratio } \\
(\mathrm{dB})\end{array}$ & $\begin{array}{c}\text { Tilt angle } \\
(\mathrm{o})\end{array}$ & $\begin{array}{c}\text { Direction } \\
\text { of polarization }\end{array}$ \\
\hline 12.0 & $6.21 \pm 0.11$ & $55.6 \pm 2$ & $89.5 \pm 0.2$ & Left \\
14.0 & $6.50 \pm 0.11$ & $57.6 \pm 2$ & $89.1 \pm 0.2$ & Left \\
16.0 & $7.02 \pm 0.11$ & $62.5 \pm 2$ & $89.2 \pm 0.2$ & Left \\
\hline
\end{tabular}

1 they increase the signal-noise ratio, because the nonadaptability is decreased (contrary to measurement in distant zone, because the existing amount of energy must cover larger area).

2 they filtrate the field in advance and by doing so (according to the Nyguist sampling theory) they increasing the distance of the monitoring/scanning that would otherwise be half of the wavelength (it is sufficient to sample from distance of five wavelengths or more).

3 they lower the reflection influence from the ground and surrounding objects.

4 they lower the demands on the precision setup of coordinates $x$ and $y$, because elements with high space frequencies change phase rapidly. Requests on axis remain unchanged.

There is a limitation of all measurements done by the monitoring in the near zone, and it is determined by the level of suppression of still waves influenced by multi reflections (multi-passages) relevant to the applicable configuration of the measured antenna and probe. In cases, when the measured antenna has smaller electric dimensions, it is practical to lower the multi reflections by increasing the distance between the aperture of the funnel probe and the structure of the measured antenna.

In such case, often the separating distance of 10 to 20 wavelengths will suppress the signal for second passage to level $-40 \mathrm{~dB}$. But during measurement of electrically large antennas, it has been discovered that separation of 10 wavelengths may correspond with suppression of the second passage only $25 \mathrm{~dB}$ for open rectangular funnel.

Even though there are many different methods how to solve this problem, the two most common represent the use of the average value for multi-monitoring and minimize the dimension of the probe aperture. Multimonitoring is a practical method used just for this purpose. During this method the measurement is done on several surfaces (based on the used method on flat surfaces or on cylindrical or spherical surfaces) that are $1 / 8$ of a wavelength apart. The average value is calculated from final radiation characteristics in the distant zone. This approach is very successful and it is usually integrated as an option in programmes that deal with measuring results processing. The minimization of the probe aperture lowers the dispersion-reflective surface of the used aperture structure.

\subsection{Probe with two outputs}

Using probes with two orthogonally polarized signals, to ensure monitoring/scanning of two data files during one scan, will lower the time to half, which is necessary for data collection in comparison with traditional monitoring/scanning with one output probe. This also enables us to use simpler construction because mechanism for probe rotation (around the probe axis) is not necessary. In combination with automatic measuring system, probes with two outputs enables us to utilize the apparatus to its maximum capacity. For the applicable probe it is necessary, to measure the gain on the axis, axis ratio, tilt angle and direction of rotation. Samples of gain measurements and polarization for probe with one and for probe with two outputs are shown in Tables 1 and 2 .

Required and necessary properties of probe with two orthogonally polarized outputs are

1 high polarization purity,

2 good insulation between both ports,

3 mutual and shared reference system of coordinates,

4 aperture with small dispersion value,

5 possibility to repeatedly connect the probe to the highfrequency track,

6 suitable installation of connection.

Probes with two outputs use square or circular waveguides with dielectrical filling. This approach however, worsens the adaptability of the probe aperture and requires careful inspection of the dielectrical material in order to find out clear and symmetrical emitting characteristics of the probe. Another option is to use circular waveguide with diameter above the critical value but at the same time it cannot be too big (that means higher aspects could not spread). That will minimize the aperture dimension and relevant wave reflection but at the same time it leads to somewhat narrow frequency band. As a sample, we may use a probe that is designed for frequencies from 5.25 to $5.35 \mathrm{GHz}$ with diameter of circular waveguide $41.9 \mathrm{~mm}$, when the low working frequency is 1.25 fold of the critical frequency for aspect TE11 and the upper working frequency is 0.78 fold of the critical frequency for aspect TE21 and 0.98 fold of the critical frequency for aspect $\mathrm{TM}_{01}$. The nominal dimension of the wall thickness of the waveguide of $3.175 \mathrm{~mm}$ is narrowed down to only $1.016 \mathrm{~mm}$ in the aperture in order to further minimize the reflection surface. The probe assembly has been calibrated by the National Institute of Standards and Technology (NIST) in USA.

This report gives you essential information, necessary for probe selection used for measurements in the near zone. Here you may find basic requirements for probes with higher gains, which may only be used for calculations of smaller angular range (eg 5$)$. However, for these types of probes you have to use probe corrections. Using probe with two outputs of orthogonally polarized signals for monitoring/scanning of two data files during one scan, lowers the time needed for data collection in half, in comparison with traditional monitoring/scanning done 
Table 2. Axis measuring results of a typical probe with two outputs

\begin{tabular}{cccccccc}
\hline $\begin{array}{c}\text { Frequency } \\
(\mathrm{GHz})\end{array}$ & Port & $\begin{array}{c}\text { Gain } \\
(\mathrm{dB})\end{array}$ & $\begin{array}{c}\text { Axis ratio } \\
(\mathrm{dB})\end{array}$ & $\begin{array}{c}\text { Tilt angle } \\
\left({ }^{\circ}\right)\end{array}$ & $\begin{array}{c}\text { Direction } \\
\text { of polarization }\end{array}$ & $\begin{array}{c}\text { Reflection } \\
\text { Amplitude }\end{array}$ & $\begin{array}{c}\text { Coeff. } \\
\text { Phase }\left({ }^{\circ}\right)\end{array}$ \\
\hline 12.0 & $\mathrm{X}$ & $16.42 \pm 0.11$ & $24.8 \pm 2$ & $-0.5 \pm 0.2$ & Left & 0.492 & -47.1 \\
14.0 & $\mathrm{X}$ & $16.41 \pm 0.11$ & $24.1 \pm 2$ & $0.0 \pm 0.2$ & Left & 0.464 & -69.5 \\
16.0 & $\mathrm{X}$ & $16.65 \pm 0.11$ & $23.5 \pm 2$ & $-0.5 \pm 0.2$ & Left & 0.513 & -85.9 \\
12.0 & $\mathrm{Y}$ & $16.65 \pm 0.11$ & $38.5 \pm 2$ & $90.7 \pm 0.2$ & Right & 0.484 & -44.7 \\
14.0 & $\mathrm{Y}$ & $16.50 \pm 0.11$ & $34.7 \pm 2$ & $90.7 \pm 0.2$ & Right & 0.460 & -67.3 \\
16.0 & $\mathrm{Y}$ & $16.63 \pm 0.11$ & $32.6 \pm 2$ & $90.4 \pm 0.2$ & Right & 0.513 & -84.0 \\
\hline
\end{tabular}

with probe with one output only. This also enables us to use simpler construction, because mechanism for probe rotation (around the probe axis by $90^{\circ}$ ) is not necessary. In combination with automatic measuring system, probes with two outputs enables us to utilize the apparatus to its maximum capacity. The insulation between ports should be better than $20 \mathrm{~dB}$. Insufficient insulation between ports $\mathrm{X}$ and $\mathrm{Y}$ causes that the measuring process depends very much on reflection endings for orthogonal port (if you using two channel receiver with input impedance of this receiver). As seen from the published specifications, the insulation is usually better than $40 \mathrm{~dB}$.

\section{CONCLUSION}

The insulation between ports should be better than $20 \mathrm{~dB}$. Insufficient insulation between ports $\mathrm{X}$ and $\mathrm{Y}$ causes that the measuring process depends very much on reflection endings for orthogonal port (if you using two channel receiver with input impedance of this receiver). As seen from the published specifications, the insulation is usually better than $40 \mathrm{~dB}$. However, it is necessary to ensure that during the use of the probe, the endings of outputs do not differ significantly from the ending during the measurement.

The study is aimed at providing an overview of the theory of near-zone measurement. The paper therefore synoptically presents detailed theoretical relationships that are crucial for the numerical processing of the near-zone measurement (field transformation from the near to distant zone). It presents relationships that are essential for the numerical processing of the near-zone measurement at the transformation of the measured field to aerial surface as well as during the computation of the gain of aerial system.

\section{Acknowledgement}

The paper was supported by project CZ.1.07/2.3.00/30.0035.

\section{REFERENCES}

[1] VACULÍKOVÁ, P.—VACULÍK, E. : Elektromagnetická kompatibilita (Electromagnetic Compatibility), Grada Publishing, Praha, 1998. (in Czech)

[2] SVAČINA, J.: Základy elektromagnetické kompatibility (Electromagnetic Compatibility Fundamentals), VUT Brno, 2001.

[3] REPJAR, A. G.-NEWELL, A. C.-FRANCIS, M. H.: Accurate Determination of Planar Near-Field Correction Parameters for Linearly Polarized Probes, IEEE Trans. Antennas Prop. AP-36 No. 6, 855-768.

Received 28 February 2014

Ján Ivanka (Ing) was born in Púchov, Slovak Republic in 1960. He received the Master degree in Military Communication and Connection Technology at the Military Academy of Technology in Brno, Czech Republic in 1984. He has joined the Department of Security Engineering at the Bata University in Zlín since 2003. His main research interests include aspects of electromagnetic interference issues and the electromagnetic susceptibility of mechatronic systems and other activities are focused on software issues designed to determine the time of death of biological material in the algor and rigor mortis processes.

Hana Chudá (Mgr, PhD) was born in Uherské Hradiště, Czech Republic in 1979. She received the Master degree in Mathematics and Physics at the Palacký University, Olomouc, Czech Republic in 2002 and the PhD degree at the same university in 2011. She has joined the Department of Mathematics at the Bata University in Zlín and she has been working as a Research Worker at this university since 2013. She main research interests include aspects theory of optical activity of crystals and theory of diffeomorfisms and automorfisms on Riemannian manifolds. 